\title{
Geographisches Gezwitscher - Analyse von Twitter-Daten als Methode im GW-Unterricht
}

\author{
" ninabrendel@uni-potsdam.de, Institut für Umweltwissenschaften und Geographie, Universität Potsdam ( $\square$ korresp. Autorin) \\ ** nils.matzner@tum.de, Department of Science, Technology \& Society, Technische Universität München \\ *** max-peter.menzel@aau.at, Institut für Geographie und Regionalforschung, Universität Klagenfurt
}

eingereicht am: 04.07.2021, akzeptiert am: 16.09.2021

Soziale Medien sind ein wesentlicher Bestandteil des Alltags von Schüler*innen und gleichzeitig zunehmend wichtig in Wirtschaft, Politik und Wissenschaft. Am Beispiel von Twitter zeigt dieser Beitrag, dass soziale Medien im Unterricht auch für die Beantwortung geographischer Fragestellungen verwendet werden können. Hierfür eignen sich Twitter-Daten aufgrund ihrer Georeferenzierung und weiterer interessanter Inhalte besonders. Der Beitrag gibt einen Überblick über die Verwendung von Twitter für sozialwissenschaftliche und humangeographische Fragestellungen und reflektiert die Nutzung von Twitter im Unterricht. Für die Unterrichtspraxis werden Beispiele zu den Themen Braunkohle, Flutereignisse und Raumwahrnehmungen sowie Anleitungen zur Auswertung, Anwendung und Reflexion von Twitter-Analysen vorgestellt.

Keywords: Twitter, soziale Medien, Forschungsmethodik, Unterrichtsmethoden

\section{Geographic tweeting - analysis of twitter data as a teaching method in geography education}

Social media are an essential part of students' everyday lives and increasingly important in economy, politics and research. Using Twitter as an example, this article shows that social media can also be used in the classroom to answer geographic questions. Twitter data is particularly suitable for this purpose because of its georeferencing and more interesting content. The article gives an overview of the use of Twitter for social science and human geography, and reflects on the use of Twitter in the classroom. Examples on the topics of lignite, flood events and spatial perceptions as well as instructions for the evaluation, application, and reflection of Twitter analyses are provided.

Keywords: Twitter, social media, research methods, teaching methods

\section{$1 \quad$ Einleitung}

Soziale Medien bestimmen zunehmend unseren Alltag, wie wir zum Beispiel mit Freund*innen kommunizieren, Nachrichten lesen und Kaufentscheidungen fällen. Ein wachsender Teil der Kommunikation über Alltägliches, Nachrichten, Wirtschaft, Politik und Forschung findet in den sozialen Medien statt. Auch wenn Facebook mit fast drei Milliarden monatlichen Nutzer*innen das Feld deutlich anführt, ziehen andere soziale Medien nach und bieten neue, attraktive Formate wie die Nutzer*innen-Inhalte dargeboten und sortiert werden. In Deutschland, was sich deutlich von anderen Ländern unterscheidet, führen YouTube und Facebook die Liste der aktiven Nutzer*innen an, während Twitter auf Platz 5 und TikTok nur auf Platz 10 rangiert (Statista 2020; Pew Research Center 2021). Die wichtige Rolle von sozialen Medien für sozialen Wandel zeigt sich unter anderem in globalen Protesten gegen Sexismus (wie bei \#metoo und \#Aufschrei; Antonakis-Nashif 2015; Xiong et al. 2019), in der Möglichkeit, Infektionsverläufe anhand von Medienverhalten zu überwachen (Chen, Lerman et al. 2020), schwer zu regulierendem Hatespeech (auch auf TikTok, Weimann \& Masri 2020), Kommunikationsblasen (Burns \& Burgess 2015) oder der Verbreitung von Rechtsextremismus (Wiertz \& Schopper 2019, 2021). In der sozialwissenschaftlichen und geographischen Forschung werden aufgrund der Verfügbarkeit großer Datenmengen Netzwerkbeziehungen, Trends verschiedener Themen, lokale Entwicklung von Diskursen (durch „geocodes“) und geographische Er- 
eignisse (beispielsweise "\#sturmflut") untersucht. Insbesondere Twitter bietet eine große Menge frei verfügbarer Daten (Stichwort: Big Data) und einfach zu bedienender Werkzeuge, um Trends und Inhalte zu veranschaulichen (Ilieva \& McPhearson 2018; Pfaffenberger 2016). Auch für Anwender*innen ohne Programmierkenntnisse lassen sich dabei interessante Betrachtungen von sozialen Medieninhalten anstellen.

Schon seit fast 15 Jahren wird der Alltag von Jugendlichen zunehmend von sozialen Medien bestimmt und diese wirken sich erheblich auf ihre Sozialisationsprozesse und Identitätsfindung aus (boyd 2008; McConnell et al. 2017). Umso wichtiger ist es, dass Kinder und Jugendliche im Unterricht einen kompetenten Umgang mit digitalen Medien erlernen. Von Seiten der Schule und der Eltern herrscht weiterhin die Sorge vor, dass Schüler*innen mit nicht kindund jugendgerechten Inhalten konfrontiert werden oder ein Suchtverhalten an den Tag legen. Da „Fake News" und manipulierte Medieninhalte die öffentlichen Debatten um soziale Medien bestimmen, ist dieser problematisierende Zugang nicht verwunderlich (Vosoughi et al. 2018).

Wir wollen einen kritischen Überblick zu Twitter im Geographieunterricht geben und Möglichkeiten und Grenzen aufzeigen. Ziel dieses Beitrages ist es, den Leser*innen Unterscheidungskriterien an die Hand zu geben, damit sie sinnvolle, aufschlussreiche und einfache Untersuchungen von Twitter von weniger sinnvollen unterscheiden können und wie sie diese positiven Anwendungsformen im Geographieunterricht einsetzen können.

Der zweite Abschnitt dieses Beitrages erläutert die sozialwissenschaftliche und geographische Forschung zu Twitter. In diesem Abschnitt gehen wir auf den Unterschied von Twitter zu anderen sozialen Medien ein, erläutern Möglichkeiten zur Datenerhebung und -auswertung und beschreiben vier zentrale Felder in der Forschung zu Twitter aus den Sozialwissenschaften und der Geographie.

Im dritten Abschnitt stellen wir die Möglichkeiten und Herausforderungen von Twitter im Geographieunterricht vor. Darin diskutieren wir den Kompetenzerwerb mittels Twitter im Geographieunterricht, verschiedene Einsatzmöglichkeiten und schließen Unterrichtsbeispiele an, welche auf die Materialien im Anhang verweisen. Anschließend resümieren wir Grenzen und Herausforderung in der Arbeit mit Twitter und ziehen ein Fazit.

\section{Twitter in der Forschung}

\subsection{Unterschiede zu anderen sozialen Medien}

Alle sozialen Medien vereint, dass sie digitale Objekte erzeugen (Rogers 2013). Dabei haben die einzelnen
Plattformen ihre eigene Technik und Medienlogik. Hashtags bei Twitter, Instagram und TikTok ermöglichen die Vernetzung von Themen und sogar eigene Öffentlichkeiten zu aktuellen Problemen (AntonakisNashif 2015). Die frühere Plattform MySpace und das heutige Facebook bieten Raum zum Erfahren der eigenen Persönlichkeit in der Öffentlichkeit, was insbesondere für Jugendliche eine große Herausforderung darstellt (boyd 2008; Good 2013). Ein Großteil von sozialwissenschaftlicher und geographischer, aber auch mathematischer und ökonomischer Forschung von sozialen Medien bezieht sich auf Twitter. Wesentliche Gründe für die Forschung mit Twitter-Daten sind der freie Zugriff auf viele Live-Daten, darüber hinaus die kostenpflichtige Verfügbarkeit nahezu aller Daten, gut nachverfolgbare Hashtags, der Anteil textueller Daten und Metadaten wie Geocodes. Im Vergleich dazu liefert Facebook nur Daten seiner Seiten („pages“) und YouTube nur von Kanälen. Einige Plattformen wie Instagram sperren jede Form von automatisiertem Zugriff auf ihre Daten.

\subsection{Datenerhebung und Auswertung}

Die Verfügbarkeit von großen nutzer*innengenerierten Datenmengen aus den sozialen Medien bietet neue Möglichkeiten, die Geographie sozialer Phänomene für den Unterricht sichtbar zu machen. Prinzipiell lässt sich nicht nur ein Ausschnitt von Daten analysieren, wie bei einer repräsentativen Umfrage, sondern gleich alle in sozialen Medien vorhandenen Daten (Bone et al. 2016). Allerdings sind bei verschiedenen Plattformen unterschiedliche Datenformen und -mengen abrufbar. Der folgende Überblick soll insbesondere die Datenerhebung und -auswertung für Twitter erläutern.

Ein grundlegendes Verständnis der Twitter-Daten ist hilfreich für deren spätere Interpretation. Über die freie Schnittstelle (API), welche Einarbeitung erfordert, lässt sich eine Tabelle mit unter anderem folgenden Daten herunterladen: Name des*der User*in (screenName), Datum und Uhrzeit des Tweets, Tweet-Text, ob es ein Retweet ist, wenn ja, von wem der Tweet geteilt wurde, die Anzahl erhaltener Tweets sowie Längen- und Breitengrad des Tweets (Pfaffenberger 2016). Auch die geteilten Inhalte, wie Bilder, Videos und URLs können ausgelesen werden. Darüber hinaus lassen sich Profildaten der User*innen abrufen, wie etwa Name, Profilbild, Selbstbeschreibungstext („bio“), gefolgte User*innen und Follower*innen. Aus diesen Daten lässt sich vieles ableiten. Beispielsweise erlaubt die Sprache der Tweets zumindest eine grobe regionale Zuordnung oder die Selbstbeschreibung im Profil eine Einschätzung der politischen Einstellung. 
Grundsätzlich sind eine große Menge Twitter-Daten verfügbar. Mit 6000 Tweets pro Sekunde und 500 Millionen Tweets pro Tag kommt man schnell in den Bereich von Big Data ${ }^{1}$. Für die Anwendbarkeit im Unterricht empfiehlt sich daher, aufgrund der besseren Übersichtlichkeit, einen Ausschnitt zu bilden (Bone et al. 2016). Hierzu bieten sich vorstrukturierte Archive an. Tweets können dazu manuell oder über die Verwendung der erweiterten Suchfunktionen, die im Folgenden beschrieben werden, eingegrenzt werden.

Datenarchive und die freie Twitter-Schnittstelle haben Vorteile und Grenzen. Die freie Schnittstelle liefert Live-Daten, ist aber auf eine Abfrage von maximal 10 Tage retrospektiv und maximal 10000 Tweets limitiert. Des Weiteren bieten viele freie Archive zu verschiedenen Themen, insbesondere zu edukativen Zwecken, frei herunterladbare Testdatensätze ${ }^{2}$. Auch bieten Forschungseinrichtungen oft Beispieldatensätze $^{3}$ an. Sollen die Positionsdaten von Tweets (Georeferenzen) genutzt werden, dann muss berücksichtigt werden, dass nur $1-3 \%$ aller Daten georeferenziert sind (Hochmair \& Cvetojevic 2014).

\subsection{Sozialwissenschaftliche und geographische Forschungsperspektiven}

Zur Auswertung der Daten wird eine Vielzahl von sozialwissenschaftlichen Methoden verwendet. Die Verfügbarkeit von großen Datenmengen verleitet zu quantitativen Analysen, aber es sollte in Forschung und Unterricht nicht „automatisch“ an quantitative Analysen gedacht werden, sondern auch an die Bedeutung von Bildern, die individuelle Identitätskonstruktion oder andere qualitativ erfassbare Phänomene. Im Folgenden werden vier Perspektiven der TwitterForschung sowie deren geographischen Ausprägungen erläutert.

Erstens werden Twitter-Praktiken untersucht, welche in den ersten Jahren als reines Friend-Following oder Verfolgen von Live-Events auftraten (Rogers 2013), sich aber später vielmehr differenzierten. Kinder und Jugendliche nutzen selbst soziale Medien nicht notwendigerweise in der gleichen Weise wie Unternehmen, Forschungseinrichtungen und staatliche Stellen. Gleichzeitig sind aber Kinder und Jugendliche, obwohl sie mit sinkender Zahl an Twitter interessiert sind (siehe unten), Zielgruppe von Werbung, Forschung und politischer Information auf dieser Plattform. Auch ist die Nutzung von Twitter geographisch ungleich verteilt. Hecht und Stephens (2014)

\footnotetext{
https://www.internetlivestats.com/twitter-statistics/

bspw. https://lionbridge.ai/datasets/top-20-twitter-datasets-fornatural-language-processing-and-machine-learning/

3 bspw. https://snap.stanford.edu/data/
}

zeigen zum Beispiel, dass Twitter-Nutzer*innen vor allem in Städten leben und daher urbane Perspektiven in den entsprechenden Diskursen dominieren.

Zweitens sind die Inhalte der Tweets, i. e. deren Text, Bilder (darunter sog. „Memes"), Videos (darunter GIFs) und Links für die Forschung interessant. Die einfachste Herangehensweise an die Tweet-Texte sind Häufigkeitsverteilungen und gemeinsames Auftreten von Wörtern oder Hashtags, beispielsweise wie oft \#metoo zu einer bestimmten Zeit vorkommt (Xiong et al. 2019). Eine der beliebtesten Methoden ist die Sentiment-Analyse, bei welcher positive und negative Konnotationen der Texte analysiert und somit Meinungen statistisch aufgedeckt werden, wie etwa das Vorkommen einer positiven Konnotation mit einer Künstlerin oder einer Stadt (Giachanou \& Crestani 2016). Mit einem an der Diskursanalyse angelehnten Verfahren hat Antonakis-Sahif (2015) gezeigt, wie private Erfahrungen sexueller Übergriffe durch den Hashtag \#Aufschrei in die öffentliche Debatte gebracht wurden.

Diskurse auf Twitter können auch aus einer geographischen Perspektive untersucht werden. Alam (2020) untersucht beispielsweise Diskurse im Bereich Umweltaktivismus an einem bestimmten Ort, nämlich West Java. Um Muster der Stigmatisierung herauszuarbeiten, untersuchen Butler et al. (2018) die Assoziationen von Orten mit bestimmten Begrifflichkeiten, etwa für welche Orte der Begriff „shithole“ verwendet wird und von welchen Orten diese Tweets gesendet werden. Solche Untersuchungen verbinden Twitter-Daten oft mit weiteren Datensätzen, um Zusammenhänge zwischen virtuellen Diskursen und der sozialen Lebenswelt der Twitter-Nutzer*innen herauszuarbeiten. Ngyuen et al. (2016) zum Beispiel verbanden Tweets mit der räumlichen Sozialstruktur und fanden am Beispiel dreier US-amerikanischer Counties heraus, dass Tweets zu „Happiness“, „Ernährung“ und "physischer Aktivität" weniger in ökonomisch benachteiligten Gebieten vorkamen.

Drittens sind Strukturmerkmale von TwitterDatensätzen wichtige Indikatoren. Hier spielen Netzwerkanalysen eine besondere Bedeutung, weil einerseits Soziale Medien einen inhärenten Netzwerkcharakter haben und andererseits Netzwerkbetrachtungen die Beziehungen zwischen User*innen, Gruppen oder Themen ermöglichen. Mittels einer Analyse von der Nachrichtendiffusion auf Twitter stellen Alperin et al. (2019) heraus, dass wissenschaftliche Artikel sich kaum aus den wissenschaftlichen Kreisen herausbewegen. Weitgehend bekannt ist das Phänomen, dass wenige User*innen viel rezipiert werden (sogenannte Influencer*innen) und viele User*innen kaum Reaktionen bekommen (Bodrunova \& Blekanov 2018). 
Netzwerkanalysen werden auch für die Beantwortung geographischer Fragestellungen verwendet. Jiang et al. (2019) untersuchten etwa den Zusammenhang zwischen einem Aufruf zur Evakuierung vor einem Hurricane und der tatsächlichen Evakuierungsentscheidung von Personen. Sie erstellten hierzu Netzwerke auf Basis von Twitter-Daten und kamen zu dem Ergebnis, dass in der Regel dem Evakuierungsaufruf gefolgt wurde, wenn auch die auf Twitter verbundenen Personen dem Aufruf gefolgt sind.

Viertens erlauben Metadaten, wie Zeit, Georeferenz oder Absendegerät (Mobiltelefon oder Desktop PC) weitere Analysen. Am weitesten verbreitet sind Zeitreihen, wie beispielsweise die Entwicklung von Debatten oder das Live-Verfolgen von Events. Gerade diese Daten werden auch für geographische Analysen verwendet, insbesondere um raum-zeitliche Muster herauszuarbeiten. So verwenden Bruijn et al. (2019) georeferenzierte Twitter-Daten zur Analyse von Flutereignissen. Ein weiteres häufiges Beispiel sind Bewegungsmuster. Hierbei werden der Ort und der Zeitpunkt des versendeten Tweets als Analysebasis verwendet. Beispiele hierfür sind die Studien von Longley et al. (2017) über die Bevölkerungsmobilität zu unterschiedlichen Tages- und Wochenzeiten in London und jene von Van Meeteren und Poorthuis (2018), welche diese Daten nutzen, um die Theorie der Zentralen Orte von Christaller zu testen. Für solche Analysen können auch die Profildaten der Twitter-Nutzer*innen hinzugezogen werden, um etwa Mobilitätsmuster nach Alter oder Geschlecht herauszuarbeiten (Luo et al. 2016). Andere Studien untersuchen etwa das Bewegungsmuster als Reaktion auf ein bestimmtes Ereignis, wie etwa Verbrechen (Lampoltshammer et al. 2014).

Im folgenden Kapitel soll nun aufgezeigt werden, wie Twitter-Analysen als wissenschaftliche Forschungsmethode auf den GW-Unterricht übertragen werden können.

\section{Twitter im Geographieunterricht}

\subsection{Kompetenzförderung mit Twitter in einem lerner*innenzentrierten Geographieunterricht}

Während die Analyse von Twitter-Daten in den Sozialwissenschaften und der Geographie bereits eine etablierte Forschungsmethodik darstellt, wird sie in der geographiedidaktischen Forschung oder als Unterrichtsmethode im Geographieunterricht bislang nur selten genutzt-zuUnrecht, wie wirhierzeigen möchten.

Um Twitter-Analysen als wissenschaftliche Methode - wie oben diskutiert - auf den GW-Unterricht zu übertragen, werden drei verschiedene Zugänge angeboten: Erstens soll aufgezeigt werden, was das Medium Twitter vor dem Hintergrund einer digitalen Transformation von Schule und Unterricht zur Förderung (fachspezifischer) Medienkompetenzen beitragen kann. Zweitens stellen wir einen methodischen Leitfaden vor (Material 1), der die Forschungsmethode für eine Anwendung im Unterrichtskontext adaptiert. Ein dritter Zugang erfolgt über die Unterrichtsinhalte, wenn anhand dreier Beispielthemen aufgezeigt wird, wie Twitter-Analysen konkret im GW-Unterricht eingesetzt werden können.

Seit mit dem Web 2.0 die Abgrenzung von Konsument*innen und Produzent*innen überwunden wurde, ist jede* $r$ in der Lage, als sogenannte Prosument*in an global im Netz geführten Diskussionen teilzuhaben (Süss et al. 2018). Dies schafft einerseits neue Beteiligungsmöglichkeiten und eine Demokratisierung von Informationen und Kommunikationsprozessen. Andererseits erfordert es von Nutzer*innen ein hohes Maß an Medienkompetenzen - die Kinder und Jugendliche durch den Unterricht ausbilden sollen. Für Österreich weist das Kompetenzmodell digi.komp informatische Kompetenzen für alle Schulstufen aus (vgl. BMBWF). Bereits in der Volksschule sollen Kinder lernen, ihr „digitales Ich im Web [zu] gestalten“, in der Mittelstufe bildet der Kompetenzbereich „Digitale Kommunikation und Social Media" ein wesentliches Element der informatischen Bildung (BMBWF). In Deutschland führt die „Strategie für Bildung in der digitalen Welt" (KMK 2017) ebenfalls (Teil-)Kompetenzbereiche an, die für Reflexion der Mediennutzung und eigenes Handeln im globalen und medialen Kontext sensibilisieren (z. B. „eigene Defizite ermitteln und nach Lösungen suchen “, „Potenziale der Digitalisierung im Sinne sozialer Integration und sozialer Teilhabe erkennen, analysieren und reflektieren“, „Interessengeleitete Setzung, Verbreitung und Dominanz von Themen in digitalen Umgebungen erkennen und beurteilen") (ebd.: 18 f.).

Anhand der Kompetenzformulierungen beider Länder wird deutlich: Es geht nicht um temporäre Umgangsstrategien mit trendigen Technologien. Ziel ist es vielmehr, Kompetenzen zu entwickeln, die Lernende auf die Herausforderungen einer digital transformierten Gesellschaft vorbereiten.

Dazu ist laut KMK-Strategie allerdings auch eine Veränderung der Lernkultur und der Lehrkräfterolle vonnöten: Lernen soll prozess- und ergebnisorientiert gestaltet sein, Lehrkräfte nehmen die Rolle von Lernbegleiter*innen ein, die ,individuelle Lernarrangements $[\ldots]$ entwickeln und verfügbar machen" (KMK 2017: 13).

Die folgenden Aspekte sollen beispielhaft verdeutlichen, wie der Einsatz von Twitter im GW-Unterricht im Sinne solch einer Neuausrichtung erfolgen kann: 
- Chance für echte Partizipation und Lerner*innenzentrierung

Durch die Demokratisierung von Information in unserer heutigen Gesellschaft liegt die Informationshoheit nicht mehr bei den Lehrenden. Statt jedoch einen Kontroll(illusions)verlust (Rosa 2015) von Lehrkräften zu bedauern, sollte dies vielmehr als Chance für hohe Stufen der Partizipation (Mayrberger 2012) von Lernenden gesehen werden, bei denen Lerngegenstand, -methode und -ziel von den Schüler*innen (mit) gestaltet werden und ihre Interessen im Zentrum des Unterrichts stehen. Über ergebnisoffene Aufgabenstellungen können Lernende über Twitter z. B. eigenen Fragen bzw. Teildiskursen des Unterrichtsthemas nachgehen. Anschließend können im Unterrichtsgespräch Informationsgüte und Intention der Darstellung analysiert und die eigene Position reflektiert werden. Die Lehrkraft unterstützt durch geeignete Impulse auf Grundlage individueller Diagnose.

- Aufzeigen von Machtstrukturen und Kommunikationsblasen

Einerseits öffnet Twitter Zugänge zu globalen Diskursen, andererseits werden über dieses soziale Medium auch Diskurse in Blasen geführt - was Nutzer*innen oftmals nicht bewusst ist und Personen oder Sichtweisen aus Kommunikationen ausschließen kann. Dies kann im Kleinen schon über die konkrete Formulierung eines Hashtags passieren (z. B. \#Atomkraft vs. \#Kernkraft) oder Vorschläge trendiger Themen, die Twitter auf Grundlage der Personen vorschlägt, denen man folgt. Durch Medienbildung in der Schule soll- ten Lernende gemeinsam mit der Lehrkraft diese „Interessengeleitete Setzung, Verbreitung und Dominanz von Themen" (KMK 2017: 19) analysieren und die „[d]ie Bedeutung von digitalen Medien für die politische Meinungsbildung und Entscheidungsfindung" reflektieren (ebd.).

- Raumkonstruktion und Bewertungskompetenz im Sinne einer Bildung für nachhaltige Entwicklung

Twitter kann eine multiperspektivische Sichtweise auf Debatten eröffnen, also Impulse, Meinungen und Bewertungen einbringen, die über die eigenen Kommunikationskreise (z. B. eurozentristische Blickwinkel, dominante Meinungen in der eigenen sozialen Gruppe, etc.) hinausgehen. Dies bietet die Möglichkeit, Perspektivenwechsel anzubahnen und Bewertungen auf Grundlage verschiedener Werte und Normen zu unterscheiden (Bewertungskompetenz im Sinne einer Bildung für nachhaltige Entwicklung).

Auch Raumkonstruktionen können so hinterfragt werden: Buschbaum et al. (2017) zeigen auf, wie über die Analyse von Hashtags (z. B. \#Freude \#shoppen) gemeinsam mit Ortsangaben (z. B. Frankfurter Stadtteil \#Bockenheim) der Tweets räumliche Bedeutungszuweisungen analysiert und vor dem Hintergrund von Raumwahrnehmung und Raumkonstruktionsprozessen ausgewertet werden können. Diese Tweets bilden damit einerseits individuelle Raumwahrnehmungen bzw. -konstruktionen ab, andererseits kreieren sie sie damit auch: „Die sozialen Medien sind somit zugleich Teil der Welt wie auch ,Weltlieferanten “" (Buschbaum et al. 2017: 116).

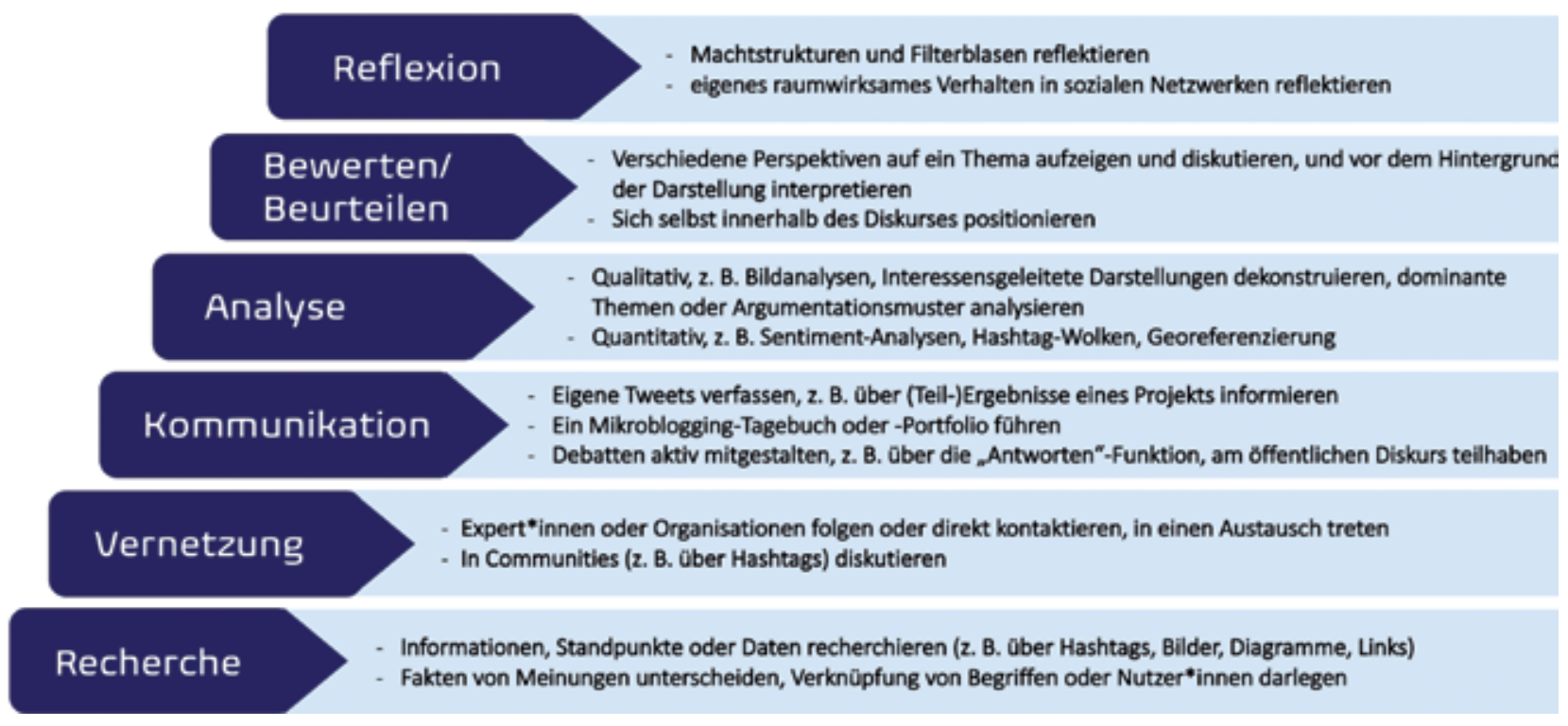

Abb. 1: Einsatzmöglichkeiten von Twitter im GW-Unterricht (eigene Darstellung) 
Welchen Beitrag die Fächer Geographie und GWUnterricht für ein Lernen in einer digitalisierten Welt leisten können, wird in einer Stellungnahme des HGD grundsätzlich dargelegt (HGD 2020).

Wie speziell Twitter im GW-Unterricht zur fachspezifischen Medienkompetenz beitragen kann, wird in Abbildung 1 exemplarisch an konkreten Einsatzszenarien erläutert. Die Kompetenzbereiche lehnen sich dabei an die oben erwähnten Medienkompetenzmodelle von Österreich und Deutschland an. Dabei empfiehlt es sich, die Kompetenzen „von unten nach oben" aufzubauen, die Bereiche sind allerdings nicht trennscharf, bedingen sich gegenseitig und sind von den spezifischen Bedingungen der Lernendengruppe abhängig zu machen.

\section{Unterrichtsbeispiel Twitter-Analyse}

Im Folgenden möchten wir aufzeigen, wie die Analyse von Twitter-Daten als (sozial- und humangeographische) Forschungsmethode auf den GW-Unterricht übertragen werden kann. Dazu bieten wir einen Leitfaden für Lehrkräfte an, der die Forschungsmethode auf den Schulkontext adaptiert und eine Schritt-fürSchritt-Anleitung zur Analyse von Twitter-Daten mit Schüler*innen bietet (siehe Anhang: Material 1): Wir orientieren uns dabei an den Schritten einer TwitterAnalyse, wie sie in der sozial- und humanwissenschaftlichen Forschung durchgeführt wird, und skizzieren, wie man in diesem Kontext eine Fragestellung definiert, die passenden Erhebungs- und Twitter-Analyse-Werkzeuge auswählt und die Daten interpretiert und reflektiert (und ggf. präsentiert). Material 2 gibt zudem eine Übersicht über Twitter-Analysewerkzeuge, die sich durch die Einfachheit der Handhabung und Visualisierung gut für den Einsatz im Unterricht eignen. Für jedes Werkzeug wird herausgestellt, für welche Art von Analysen es sich besonders gut (oder weniger) eignet und was es bei diesem Tool zu beachten gilt.

Im Folgenden stellen wir drei Unterrichtsbausteine vor, die skizzieren, auf welche Weise die forschungsmethodischen Einsatzfelder von Twitter in der Humangeographie - sinnvoll angepasst - im GW-Unterricht Einsatz finden können.

\section{Beispiel A: Analyse zum Thema Braunkohle}

Wie wird das Thema Braunkohle aktuell diskutiert? Dazu kann zunächst eine einfache Recherche über den Begriff oder den Hashtag \#Braunkohle durchgeführt und die Ergebnisse analysiert werden (siehe hierzu auch die Reflexionsimpulse in Material 3). Welche Akteur*innen äußern sich in welcher Weise? Welche Informationen werden geteilt, welche Meinungen vertreten? Auch eine Analyse der getwitterten Bilder oder Videos kann sehr aufschlussreich sein (dazu in den Ergebnissen nach „Fotos“ filtern), um verschiedene Perspektiven auf das Thema zu diskutieren.

Um einen quantitativen Überblick über die Twitter-Diskussion zu erhalten, bietet sich eine HashtagAnalyse an: Welche Begriffe werden mit dem Thema Braunkohle assoziiert? Wie ändert sich die Diskussion über Braunkohle über die Zeit? Dies kann beispielsweise mittels Wortwolken zum Begriff „Braunkohle" analysiert werden, die über die Zeitspanne einer Unterrichtsreihe zu diesem Thema erstellt werden (sucht man nur nach dem Begriff „Braunkohle“, so wird man alle Tweets zu dem Thema finden. Wenn man nach "\#Braunkohle" sucht, dann findet man die gezielte Themensetzung von Twitter-User*innen).

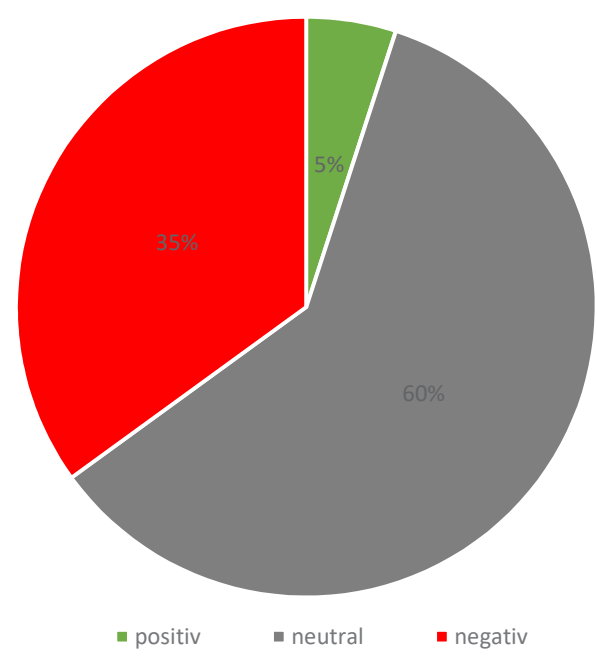

Abb. 2: Sentiment-Analyse (mit dem Begriff verbundene Gefühle) zum Begriff „Braunkohle“ (Datenquelle: social-searcher.com, eigene Darstellung)

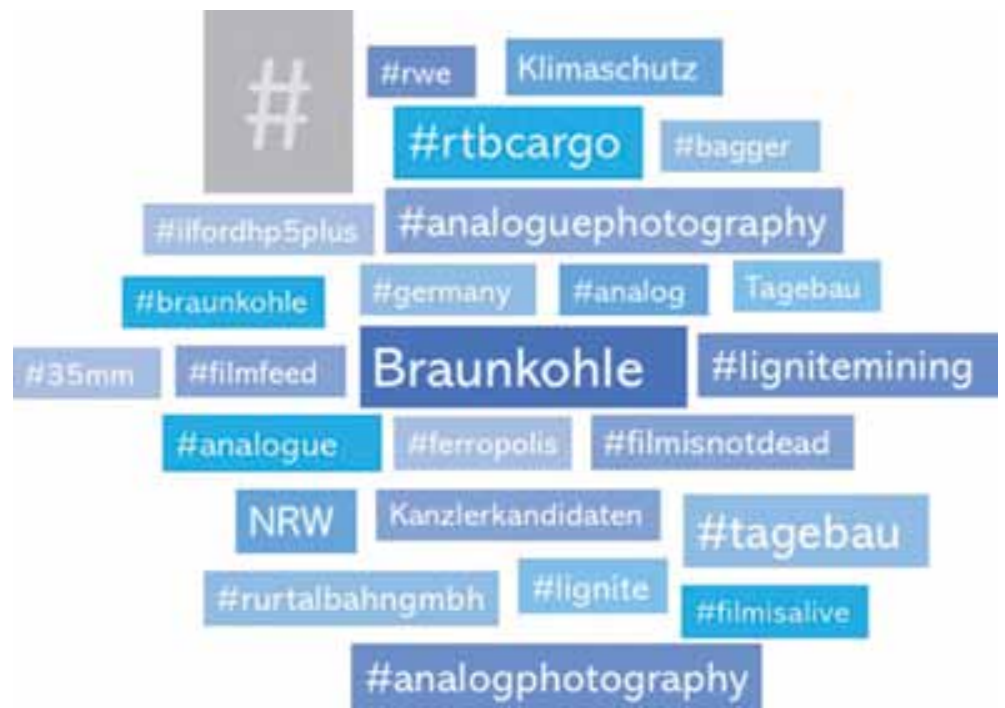

Abb. 3: Darstellung mit dem Begriff „Braunkohle“ assoziierter Begriffe und Hashtags in einer Wortwolke (Datenquelle: social-searcher.com, eigene Darstellung) 
Dies verdeutlicht, welche anderen Themen in der Twitter-Diskussion mit Braunkohle dauerhaft verbunden werden und wie sich der Diskurs durch tagesaktuelle Nachrichten oder Trends verändert (z. B. anstehende Wahlen, Protestaktionen etc.). In Abb. 2 und 3 sind Analyseergebnisse zum Zeitpunkt März 2021 abgebildet.

Hierbei wird deutlich, dass der Begriff „Braunkohle" stark mit Klimaschutz assoziiert ist; ein in diesem Zusammenhang dominanter Ort ist das Bundesland Nordrhein-Westfalen in Deutschland. Die Bezüge zu politischen Akteur*innen lässt sich mit der Bekanntgabe der Kanzlerkandidat*innen und dem damit verbundenen Start des Wahlkampfes zu diesem Zeitpunkt erklären. Zudem fallen einige Begriffe aus der Fotografie auf: Wirft man einen Blick auf die mit unter diesem Begriff getwitterten Bilder, wird klar, dass Braunkohle-Abbau mit einer gewissen Ästhetik verbunden wird (im Positiven wie auch Negativen). Eine Sentiment-Analyse klärt die allgemeine Gefühlslage zu diesem Thema: Die Tweets zu diesem Thema sind eher mit negativen als positiven Gefühlen assoziiert.

Diese Erkenntnisse können entweder zu einem bestimmten Zeitpunkt z. B. zu den Darstellungen im Schulbuch in Bezug gesetzt werden oder über den Verlauf der Unterrichtsreihe hinweg wiederholt werden und es können Veränderungen der Debatte beobachtet werden.

\section{Baustein B: Flutereignisse in Echtzeit analysieren}

Auch für Themenfelder der Physischen Geographie können Twitter-Daten auf eindrucksvolle Weise Pro- zesse verdeutlichen: Der Global Flood Monitor ${ }^{4}$ beispielsweise visualisiert Flutereignisse auf einer Weltkarte mittels Tweets in elf Sprachen - in Echtzeit. Das Projekt des Institute for Environmental Studies der Universität Amsterdam verdeutlicht einerseits Flutereignisse als Flächensignaturen, per Klick können dazu aber auch einzelne Tweets angesehen und analysiert werden. Ein Zeitregler erlaubt es zudem, die räumliche Verteilung der Flutereignisse auf der Erde in Abhängigkeit von Jahreszeiten oder außergewöhnlich regenreichen oder trockenen Perioden zu analysieren. Wichtig ist es hierbei auf Grenzen der Twitter-Analyse (siehe Kapitel 4) einzugehen: Da der Begriff \#flood auch im übertragenen Sinne verwendet wird, stehen einige Tweets nicht in Zusammenhang mit Flutereignissen. Dennoch zeigen die Autoren in ihrem dazugehörigen wissenschaftlichen Artikel auf, dass 90\% der Tweets mit tatsächlichen Flutereignissen in Verbindung stehen, die zum Teil in anderen Datenbanken nicht erfasst werden (De Bruijn et al. 2019). Hier zeigt sich das große Potential von Twitter-Daten als ergänzende Datenquelle - und zwar in einer im Unterricht sehr leicht nutzbaren Weise. Abb. 4 und 5 zeigen jeweils einen Screenshot der Anwendung.

\section{Baustein C: Raumwahrnehmung, -darstellung und -konstruktion}

Ein dritter Ansatz soll verdeutlichen, wie (unterschiedlich) Räume in sozialen Netzwerken dargestellt und so bewusst Raumvorstellungen konstruiert werden. Als Einstieg können die Schüler*innen z. B. auf-

\section{https://www.globalfloodmonitor.org}

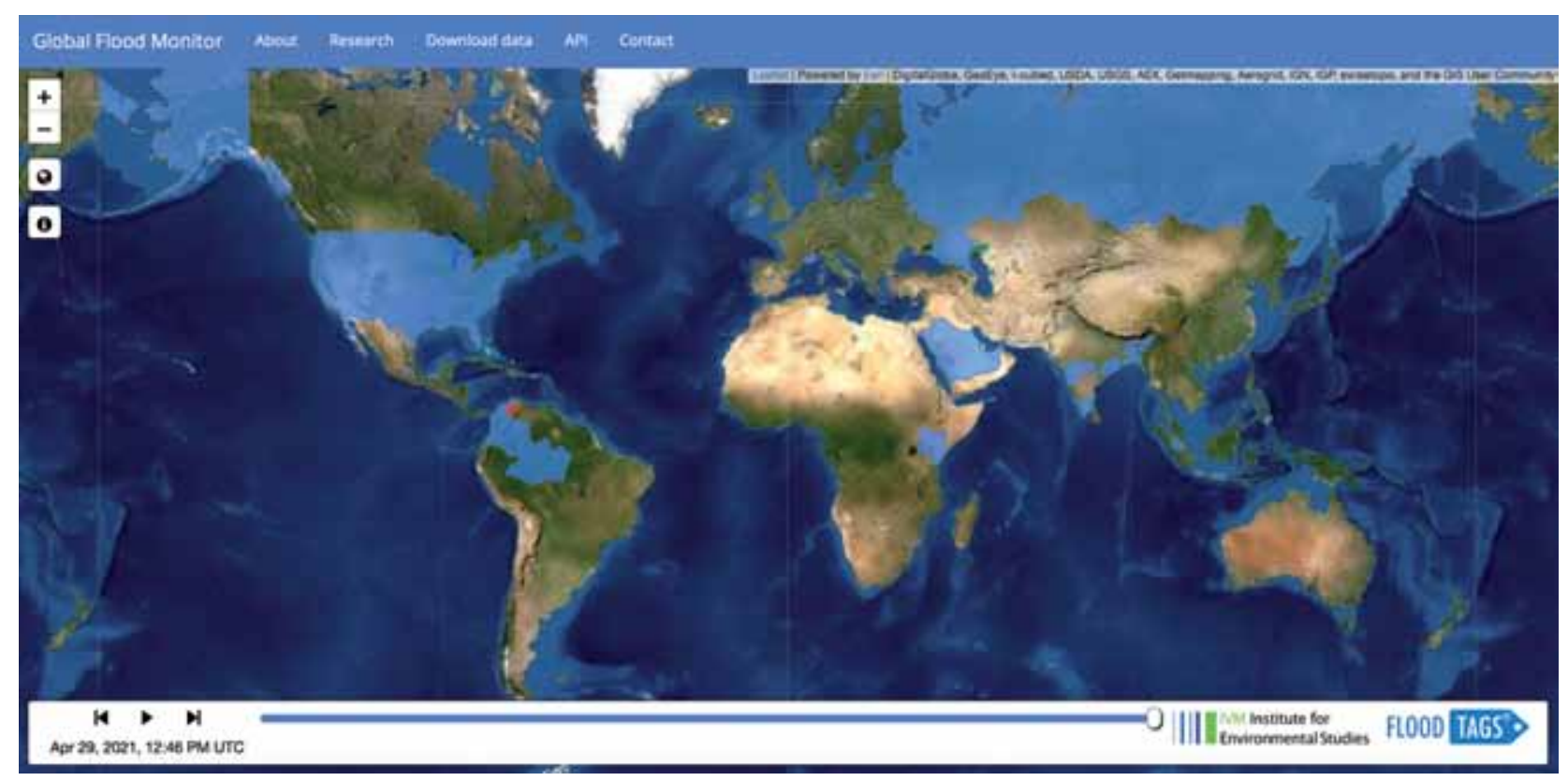

Abb. 4: Visualisierung der Flutereignisse in Echtzeit oder zu einem bestimmten Zeitpunkt über die Analyse des Begriffs „flood“ in Twitter-Nachrichten. (Quelle: Global Flood Monitor, aufgerufen am 29.04.2021) 


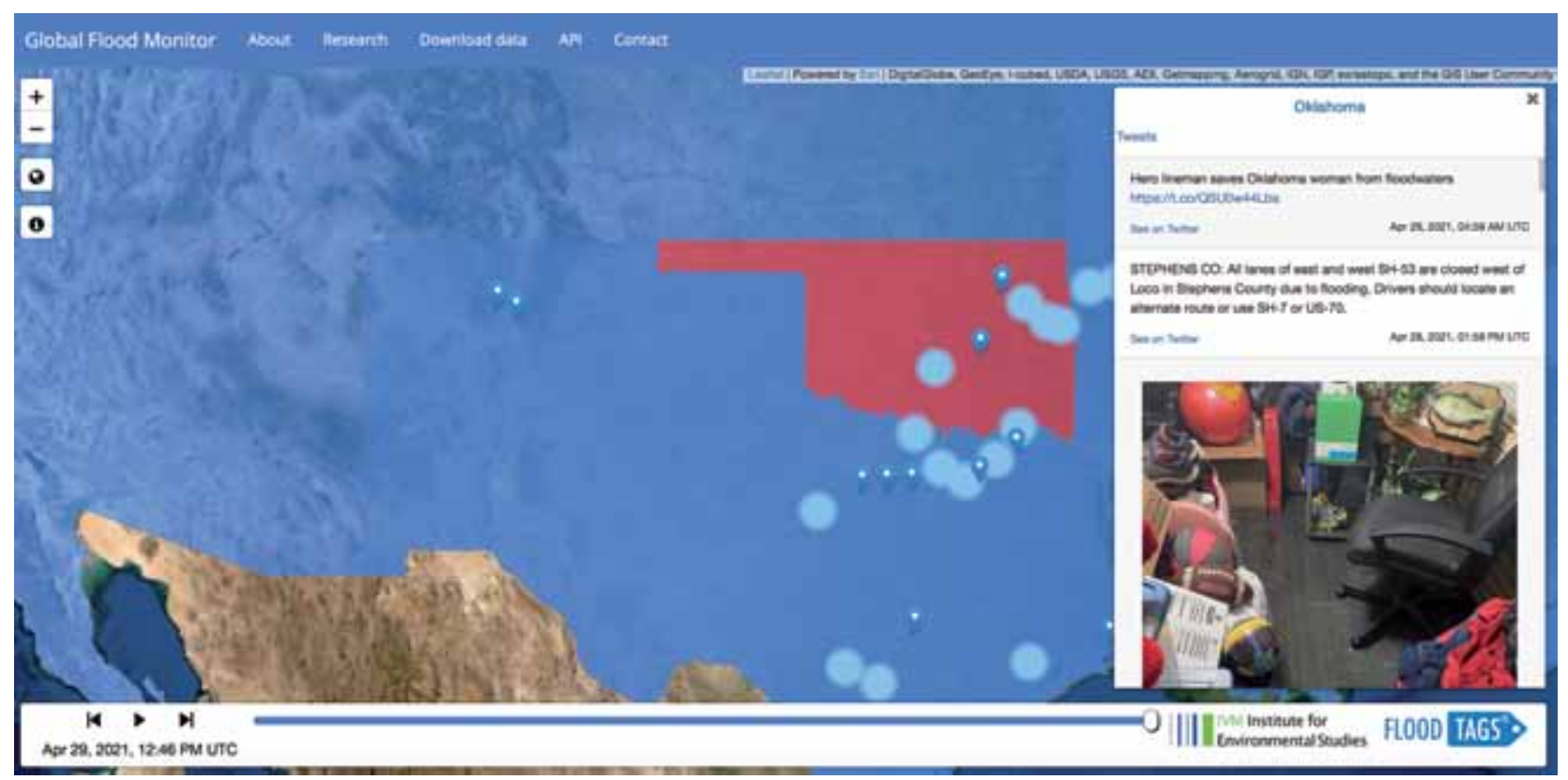

Abb. 5: Geolokalisierung einzelner Twitter-Tweets, die den Begriff "flood“ beinhalten, inklusive Detailansicht der Tweets. (Quelle: Global Flood Monitor, aufgerufen am 20.04.2021)

gefordert werden, in einer Mind-Map Assoziationen zu einer Stadt oder Region - in unserem Beispiel wählen wir Wien - festzuhalten. Anschließend werden diese persönlichen Wahrnehmungen der Stadt mit der Selbstdarstellung der Stadt auf Twitter (@Stadt-Wien) sowie der Wahrnehmung in deutschen und englischsprachigen Tweets verglichen. Dazu eignen sich neben einfachen Schlagwort- oder Hashtagsuchen auch Wortwolken, eine Analyse verknüpfter Begriffe oder die Auswertung von Twitter-Fotos. Leitende Fragen könnten sein: Wie nehmen wir die Stadt Wien wahr? Wie inszeniert sich die Stadt Wien selbst? Welche Elemente fallen in der Twitter-Debatte unter dem Hashtag \#wien auf, wie unterscheiden sie sich von den Diskussionen unter dem internationalen Hashtag \#vienna?

Die Ergebnisse können wiederum in Bezug gesetzt werden zu anderen Städten, z. B. über eine Analyse der Twitter-Beiträge zu \#graz oder \#linz. Essentiell ist es dabei, mit den Lernenden Stereotypenbildung zu diskutieren, genauso wie die Konstruktion von Räumen über Sprachmittel.

\section{Grenzen, Herausforderungen und ein Fazit}

Auch wenn Twitter und soziale Medien im Allgemeinen nicht neu sind, stellen sie doch ein moving target dar. Beobachter*innen aus der Forschung, Bildung und Erziehung sind vor ständig neue Herausforderungen gestellt, wenn neue Plattformen entstehen, sich neue Trends entfalten und sich Nutzungsweisen von Medien ändern.
Dieser Beitrag hat eine Vielzahl an sozialwissenschaftlichen Forschungsperspektiven und geographischen Themen zu Twitter angeführt. Zusammenfassend ist festzuhalten, dass georeferenzierte Tweets nur eine unter vielen Möglichkeiten ist, geographische Fragestellungen zu bearbeiten, zumal leider wenige Tweets diese Referenzen aufweisen. Auch mit Inhaltsanalysen oder der Verschränkung mit weiteren Metadaten lassen sich wichtige Schlüsse ziehen.

Ein großes Potential von Twitter liegt darin, auf einfache Weise ein Einblick in öffentliche Diskurse zu geben. Es müssen allerdings auch die Grenzen und Herausforderungen dieser Methoden reflektiert werden, um eine Twitter-Analyse sinnvoll und gewinnbringend einzusetzen: Denn die Analyse einer Twitter-Debatte bildet nur die Debatte ab, die auf Twitter geführt wird und nicht über andere Medien. Es ist also ein Ausschnitt aus einer öffentlichen Debatte, die nicht mit dem öffentlichen Diskurs gleichgesetzt werden kann.

Gleichermaßen müssen Ländervergleiche über Twitter mit Vorsicht interpretiert werden. Denn das Medium wird je nach Land unterschiedlich stark genutzt oder seine Funktion wird durch andere soziale Medien ersetzt (z. B. in China). Je nach Lokation unterscheiden sich auch die Suchergebnisse: Auf der Twitter-Startseite suggeriert die Spalte „Trends bei Twitter" zudem eine neutrale Darstellung trendiger Diskussionen. Meist werden die dort angezeigten Themen allerdings gefiltert durch Standort, Nutzer*innenprofil und Follower*innen. Ein weiterer Einflussfaktor auf die Ergebnisse ist die gewählte Sprache. So werden z. T. unterschiedliche Schlagworte verwendet (z. B. „lockdown“ im UK und in Europa, 
„stay-at-home-order" in den USA) oder die Formulierung bei der Hashtag-Suche bestimmt die inhaltliche Ausrichtung der Tweets (z. B. \#Atomkraft gegenüber \#Kernkraft).

Eine weitere Einschränkung betrifft Demo-Versionen: Diese greifen häufig nur auf eine begrenzte Anzahl von Tweets zurück und bilden damit nicht die gesamte Debatte auf Twitter ab. Aufgrund dieser kleinen Stichprobe können also keine allgemeingültigen Aussagen getroffen werden.

Alle diese Einschränkungen sind eine Herausforderung für Forschung und Unterricht zugleich. Forscher*innen müssen immer mit Unvollständigkeit umgehen und im Unterricht sollte diese ehrlich thematisiert werden.

$\mathrm{Zu}$ Guter Letzt freuen sich die Autor*innen über Feedback oder Vernetzung via Twitter (@NinaAnnaBrendel und @Motoko42).

\section{Acknowledgement}

Wir danken der Redaktion der GW-Unterricht, den zwei anonymen Reviewer*innen sowie Sabine Matzner für hilfreiche Hinweise. Zudem bedanken wir uns herzlich für die Förderung der Deutschen Forschungsgemeinschaft und dem Open Access Publishing Fonds der Universität Potsdam.

\section{$6 \quad$ Literatur}

Alperin, J. P., C. J. Gomez \& S. Haustein (2019): Identifying diffusion patterns of research articles on Twitter. A case study of online engagement with open access articles. In: Public Understand. Sci. 28(1). S. 2-18. DOI: $10.1177 / 0963662518761733$

Antonakis-Nashif, A. (2015): Hashtagging the Invisible: Bringing Private Experiences into Public Debate. In: Rambukkana, N. (Hg.): HashtagPublics. The power and politics of discursive networks. (= Digital formations, 103). Peter Lang, New York, Bern, Frankfurt, Berlin, Brussels, Vienna, Oxford, Warsaw, S. 101-113.

BMBWF (2021): digi.komp. Digitale Kompetenzen Informatische Bildung. https://digikomp.at/index. php?id=530 (09.11.2021).

Bodrunova, S. S. \& I. S. Blekanov (2018): Power Laws in Ad Hoc Conflictual Discussions on Twitter. In: Alexandrov, D. A., A. V. Boukhanovsky, A. V. Chugunov, Y. Kabanov \& O. Koltsova (Hrsg.): Digital Transformation and Global Society. Springer International Publishing, Cham (859). S. 67-82.

Bone, J., C. D. Emele, A. Abdul, G. Coghill \& W. Pang (2016): The social sciences and the web. From 'Lurking' to interdisciplinary 'Big Data' research. In: Methodological Innovations 9(2). S. 1-14. DOI: $10.1177 / 2059799116630665$ boyd, d. (2008): Why Youth (Heart) Social Network Sites: The Role of Networked Publics in Teenage Social Life. In: Buckingham, D. (Hg.): Youth, identity, and digital media. (= The John D. and Catherine T. MacArthur Foundation series on digital media and learning) MIT Press, Cambridge Mass. u. a. S. 119-142.

Burns, A. \& J. Burgess (2015): Twitter Hashtags from Ad Hoc to Calculated Publics. In: Rambukkana, N. (Hg.): HashtagPublics. The power and politics of discursive networks. (Digital formations, 103) Peter Lang, New York, Bern, Frankfurt, Berlin, Brussels, Vienna, Oxford, Warsaw. S. 13-27.

Buschbaum, K., A. Blitz, C. Reithmeier, \& D. Kanwischer (2017): Hashtags und Raumkonstruktionen: Eine explorative Studie zum Potential von digitalen Methoden zur Analyse raumzeitlicher Daten in sozialen Medien. In: gis. Science 4. S. 115-125.

Chen, E., K. Lerman \& E. Ferrara (2020): Tracking Social Media Discourse About the COVID-19 Pandemic: Development of a Public Coronavirus Twitter Data Set. In: JMIR public health and surveillance 6(2), e19273. DOI: $10.2196 / 19273$

De Bruijn, J. A., H. de Moel, B. Jongman, M. C. de Ruiter, J. Wagemaker \& J. C. H. Aerts (2019): A global database of historic and real-time flood events based on social media. In: Scientific Data 6(311). S. 1-12. https://doi.org/ doi:10.1038/s41597-019-0326-9

Giachanou, A. \& F. Crestani (2016): Like It or Not. A Survey of Twitter Sentiment Analysis Methods. In: ACM Computing Surveys 49(2). S. 1-41. DOI: 10.1145/2938640

Good, K. D. (2013): From scrapbook to Facebook: A history of personal media assemblage and archives. In: New Media \& Society 15(4). S. 557-573. DOI: $10.1177 / 1461444812458432$

Hecht, B. \& M. Stephens (2014): A tale of cities: Urban biases in volunteered geographic information. In Proceedings of the International AAAI Conference on Web and Social Media 8(1). S. 197-205.

Hochmair, H. \& S. Cvetojevic (2014): Assessing the Usability of Georeferenced Tweets for the Extraction of Travel Patterns: A Case Study for Austria and Florida. In: GI_Forum 2014 - Geospatial Innovation for Society. Austrian Academy of Sciences Press, Salzburg, Vienna. S. 30-39.

Hochschulverband für Geographiedidaktik (HGD) (2020): Der Beitrag des Fachs Geographie zur Bildung in einer durch Digitalisierung und Mediatisierung geprägten Welt. http://geographiedidaktik.org/wp-content/uploads/2020/11/Positionspapier_Geographische_Bildung_und_Digitalisierung_2020.pdf (06.09.2021).

Ilieva, R. T. \& T. McPhearson (2018): Social-media data for urban sustainability. In: Nature Sustainability 1(10). S. 553-565. DOI: 10.1038/s41893-018-0153-6

KMK (Kultusministerkonferenz, 2017): Strategie der Kultusministerkonferenz „Bildung in der digitalen Welt“. https://www.kmk.org/fileadmin/pdf/PresseUndAktuelles/2018/Digitalstrategie_2017_mit_Weiterbildung.pdf (01.05.2021). 
Mayrberger, K. (2011): Partizipatives Lernen mit dem Social Web gestalten. Zum Widerspruch einer verordneten Partizipation. In: MedienPädagogik: Zeitschrift für Theorie und Praxis der Medienbildung 21. S. 1-25.

McConnell, E. A., A. Clifford, A. K. Korpak, G. Phillips \& M. Birkett (2017): Identity, Victimization, and Support: Facebook Experiences and Mental Health Among LGBTQ Youth. In: Computers in Human Behavior 76. S. 237-244. DOI: 10.1016/j.chb.2017.07.026

Pfaffenberger, F. (2016): Twitter als Basis wissenschaftlicher Studien. Springer Fachmedien Wiesbaden, Wiesbaden.

Pew Research Center (2021): News Use Across Social Media Platforms in 2020. https://www.journalism. org/2021/01/12/news-use-across-social-media-platforms-in-2020/

Rogers, R. (2013): Debanalizing Twitter. In: Davis, H. (Hg.): Proceedings of the 5th Annual ACM Web Science Conference. Paris, France, 2/5/2013-4/5/2013. ACM Special Interest Group on Hypertext, Hypermedia, and Web. ACM, New York. S. 356-365.

Rosa, L. (2015): Verlust und Neugewinn: Lernen und Lehren im Medienumbruch. 4. Netzwerktagung „Medienkompetenz stärkt Brandenburg“ am 25. November 2015 in der Filmuniversität Babelsberg https://www.youtube. com/watch?v=GFmRsVwz-hk (01.05.2021).

Statista (2020): Social media used in Germany as of 3rd quarter 2020, by share of respondents https://www.statista.com/statistics/1059426/social-media-usage-germany/ (06.09.2021).

Statista (2021): Most popular social networks of teenagers in the United States from fall 2012 to fall 2020. https:// www.statista.com/statistics/250172/social-network-usage-of-us-teens-and-young-adults/ (01.05.2021).

Süss, D., C. Lampert \& C. W. Trültzsch-Wijnen (2018): Mediendidaktik: Lehren und Lernen mit Medien. In: Medienpädagogik: Ein Studienbuch zur Einführung. Springer VR, Wiesbaden. S. 161-188. https://doi. org/10.1007/978-3-658-19824-4_7

van Meeteren, M. \& A. Poorthuis (2018): Christaller and "big data": recalibrating central place theory via the geoweb. In: Urban Geography 39(1). S. 122-148.

Vosoughi, S., D. Roy \& S. Aral (2018): The spread of true and false news online. In: Science 359(6380). S. 1146-1151. DOI: $10.1126 /$ science.aap9559

Weimann, G. \& N. Masri (2020): Research Note: Spreading Hate on TikTok. In: Studies in Conflict \& Terrorism. S. 1-14. DOI: 10.1080/1057610X.2020.1780027

Wiertz, T. \& T. Schopper (2019): Theoretische und methodische Perspektiven für eine Diskursforschung im digitalen Raum. In: Geographische Zeitschrift 107(4). S. 254-281.

Wiertz, T. \& T. Schopper (2021): Geopolitische Leitbilder der „Neuen Rechten“ in digitalen Medien - Projektstart! https://human.geographie.uni-freiburg.de/digitalgeopolitics/projektbeginn-extreme-rechte-in-digitalen-medien/ (16.09.2021).

Xiong, Y., M. Cho \& B. Boatwright (2019): Hashtag activism and message frames among social movement organizations: Semantic network analysis and thematic analysis of Twitter during the \#MeToo movement. In: Public Relations Review 45(1). S. 10-23. DOI: 10.1016/j.pubrev.2018.10.014 


\title{
Material 1: Twitter-Daten im GW-Unterricht auswerten
}

\author{
eine Schritt-für-Schritt-Anleitung
}

\section{Schematisches Vorgehen in der Forschung}

Wie in jedem wissenschaftlichen Forschungsprozess beginnt die Analyse mit der Formulierung einer Forschungsfrage. Ausgehend von dieser Frage werden dann verschiedene Analyseschritte vollzogen, die ggf. wiederholt werden, bis die Ergebnisse die Forschungsfrage zufriedenstellend beantworten können (oder die Forschungsfrage adaptiert oder verworfen wird, wenn eine Beantwortung nicht möglich ist). Am Ende des Forschungsprozesses steht die Aufarbeitung der Ergebnisse in einer zielgruppenspezifischen Ergebnispräsentation. Abbildung 1 veranschaulicht diesen Prozess.

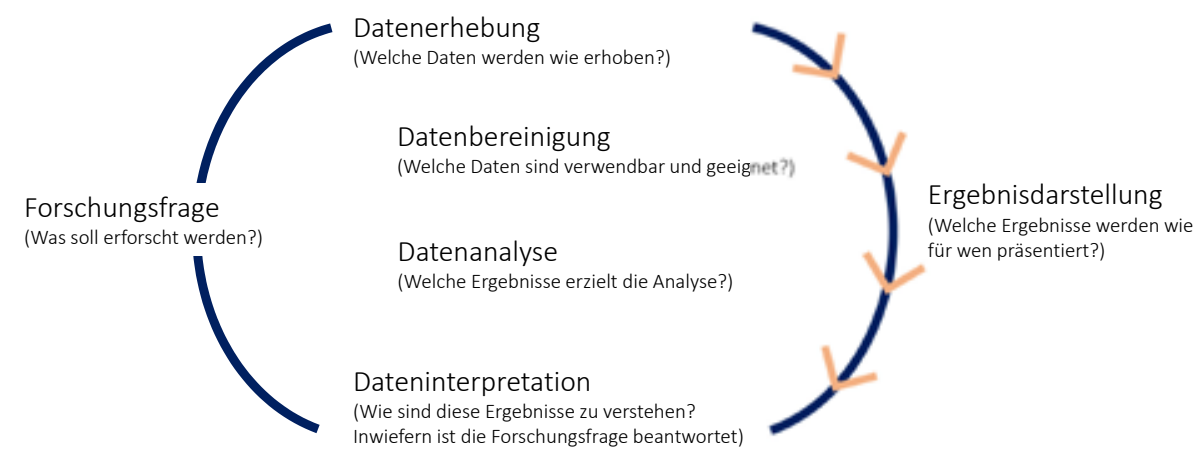

Abb. 6: Darstellung des forschungsmethodischen Analyseprozesses (eigene Darstellung)

\section{Adaptiertes Vorgehen in der Schule}

Überträgt man diesen Prozess in eine Unterrichtsmethodik, müssen mehrere Veränderungen vorgenommen werden: Zum einen muss der Prozess zeitlich deutlich verkürzt und im Umfang reduziert werden, um ihn in einer oder (wenigen) Unterrichtsstunde(n) durchzuführen. Zudem muss die Komplexität der Forschungsfrage reduziert und Analyseprozesse so vereinfacht werden, dass sie für Lernende handhabbar sind. Nichtsdestotrotz soll jedoch die Grundstruktur der Analyseschritte erhalten bleiben, um Lernenden die Grundlage des wissenschaftlichen Arbeitens zu verdeutlichen.

\section{Schritt 1: Fragestellung definieren}

Die Strukturierung ihrer Arbeit durch eine Leitfrage kennen die Schüler*innen aus dem Unterricht, der (meist) ebenfalls durch eine Frage- oder Problemstellung bestimmt wird. Die Fragestellung für einen Forschungsprozess muss noch sorgsamer formuliert und jedes Wort genau überlegt sein. Ein mögliches Vorgehen ist, mehrere Fragestellungen von den Schüler*innen vorschlagen und formulieren zu lassen, um ihre Fragen dann gemeinsam in „Forschungsfragen“ umzuformulieren. Dazu können folgende Fragen helfen:

- Trifft diese Frage den Kern dessen, was wir herausfinden möchten?

- Können wir diese Frage (mit den uns zur Verfügung stehenden Möglichkeiten) beantworten?

- Ist die Frage zu speziell oder zu allgemein formuliert?

Je nachdem, welche Frage gewählt wird, ist ggf. noch eine Reflexion ratsam, ob eine Twitter-Analyse eine geeignete und passende Methode darstellt. Wie in der Forschung auch, sollte sich die verwendete Methode immer nach der konkret formulierten Fragestellung richten.

\section{Schritt 2: Auswahl des Erhebungswerkzeugs}

Da die technischen Kompetenzen und Ressourcen im Unterricht begrenzt sind, ist es ratsam, einfache und bestenfalls visuell aufbereitete Twitter-Analysewerkzeuge zu verwenden. Einfache und kostenlose Analysewerkzeuge bieten z. B. onemilliontweetmap, OmniSci Tweet Map oder tweeplers. 
Dabei ist bei der Wahl darauf zu achten, ob eine lokale Verortung, Aussagen zur Häufigkeit (an einem bestimmten Ort), Kombination verschiedener Datenquellen (z. B. Georeferenzierung und Hashtag) o. a. im Fokus der Analyse stehen und das Werkzeug den entsprechenden Leistungsumfang bietet. Auch mehrere Werkzeuge können ergänzend verwendet werden.

\section{Schritt 3: Erhebung der Daten inkl. Überlegungen zur Stichprobe}

In diesem Schritt arbeiten die Schüler*innen in Gruppen, allein oder als Teams mit den Werkzeugen und analysieren die Twitter-Daten. Hierbei ist es wesentlich zu reflektieren, welche Eingaben zu welchen Ergebnissen führen: Welche Hashtags sollen für die Suche genutzt werden? Welche Filter werden genutzt, z. B. in Bezug auf die Datenquelle (nur Tweets oder auch Bild-Beiträge von Instagram?), die Sprache (z. B. Englisch oder Deutsch?) oder die Lokation (Deutschland, Europa oder weltweit?). Damit wird in gewisser Weise die Stichprobe festgelegt.

\section{Schritt 4: Datenanalyse}

Die Schritte 3, 4 und 5 sollten für die Lernenden deutlich voneinander abgegrenzt werden, auch wenn sie häufig parallel ablaufen. Werden in Schritt 3 nur Informationen gesammelt, werden die Ergebnisse in Schritt 4 nun ausgewertet (also objektiv verglichen, kategorisiert oder gruppiert) und erst in Schritt 5 interpretativ bewertet. Ziel von Schritt 4 ist es also, anhand der gelieferten Daten objektive, beschreibende (!) Aussagen zur Datenlage zu treffen. Dazu gehört immer auch eine Reflexion von Erhebungsfehlern oder -beeinflussungen (z. B. Ist der gewählte Hashtag objektiv oder suggestiv? Verändern sich die Ergebnisse durch andere Filter, z. B. Einbezug der englischen Sprache?)

\section{Schritt 5: Dateninterpretation}

Erst in diesem Schritt erfolgt eine Bewertung der erzielten Ergebnisse, z. B. im Hinblick auf Vorwissen der Schüler*innen, auf zusätzlich zur Verfügung gestellte Daten (z. B. Statistiken, Diagramme, Texte) oder Widersprüche, die aus den Ergebnissen hervortreten. Verschiedene Interpretationsansätze sollten unter den Schüler*innen und unter Beratung der Lehrkraft diskutiert und reflektiert werden.

\section{Schritt 6: Reflexion des Erhebungs- und Analyseprozesses}

In diesem Schritt wird das bisherige Vorgehen reflektiert und es werden daraus Schlüsse für das weitere Vorgehen gezogen. Hierbei sollten Fragen gestellt werden, wie z. B.:

- Welche Aussagekraft haben die Ergebnisse unserer Analyse?

- Wie beeinflusst unser Vorgehen die Ergebnisse, gehen wir objektiv vor oder beeinflussen wir durch Erwartungen oder einseitige Analyse die Ergebnisse?

- Können wir mit den Ergebnissen die Fragestellung (ausreichend) beantworten?

- Welche Aussagekraft haben unsere Ergebnisse?

Werden Beeinflussungen auf die Analyse festgestellt oder kommt man zu dem Schluss, dass die Fragestellung noch nicht beantwortet werden kann, kann es sinnvoll sein, einen weiteren Analysezyklus zu durchlaufen. Dazu kann die Fragestellung angepasst oder erweitert werden, vielleicht bedarf es eines anderen Analysewerkzeugs oder einer anderen Datenauswahl. Den Schüler*innen sollte bewusst werden, dass ein weiterer Durchlauf keine Schwäche im Planungsprozess bedeutet, sondern ein normaler und sogar wichtiger Teil des Forschungsprozesses.

\section{Schritt 7: Ergebnispräsentation}

Kommen die Schüler*innen zu dem Schluss, dass ihre Fragestellung mit den erhobenen Daten und der durchgeführten Analyse und Interpretation gut beantwortet werden kann, erfolgt im letzten Schritt die adressatengerechte Vermittlung ihrer Erkenntnisse. Dies kann in Form einer Präsentation (analog oder digital) geschehen und sich an die Mitschüler*innen oder an ein breiteres Publikum wenden (z. B. Veröffentlichung im Internet, Veröffentlichung der Erkenntnisse über Twitter). 


\section{Material 2: Anwendungen für die Twitter-Analyse im Unterricht}

Leistungsumfang: Beschreibt die Leistungen sowie Umfang und Tiefe der Einsatzszenarien. Die Zahl der Sterne gibt an, wie gut die Web-Anwendung aus Sicht der Autor*innen für eine Twitter-Analyse im Unterricht geeignet

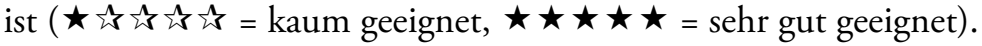

Schwierigkeitsgrad: Beschreibt die erforderlichen technischen Kenntnisse und Medienkompetenzen. Einige Anwendungen sind intuitiv bedienbar und leicht zu interpretieren $(\star \star \star \star \star)$, andere Anwendungen benötigen

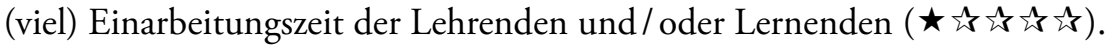

\begin{tabular}{|c|c|c|c|}
\hline Name des Werkzeugs & Internetadresse & Leistungsumfang & Schwierigkeitsgrad \\
\hline OneMillionTweetMap & $\begin{array}{l}\text { https://onemilliontweetmap. } \\
\underline{\text { com/ }}\end{array}$ & 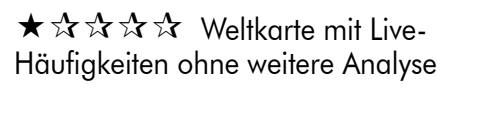 & 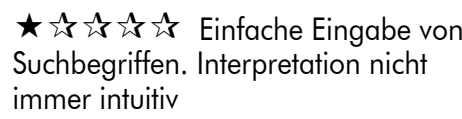 \\
\hline OmniSci Tweet Map & $\begin{array}{l}\text { https://www.omnisci.com/ } \\
\underline{\text { demos/tweetmap }}\end{array}$ & $\begin{array}{l}\star \star \star \forall \star \Delta \text { Anzeige von Top } \\
\text { Hashtags und Suchbegriffen, Fokus } \\
\text { auf Länder/Regionen und Zeiffenster. } \\
\text { Übersichtlich! }\end{array}$ & 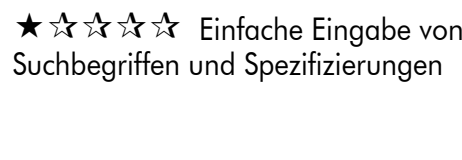 \\
\hline Global Flood Monitor & $\begin{array}{l}\text { https://www.globalfloodmo- } \\
\text { nitor.org }\end{array}$ & $\begin{array}{l}\star \star \star \forall \star \Delta \text { Übersichtlicher Zeit- } \\
\text { verlauf von "Fluten" auf der Welt- } \\
\text { karte. Detailansicht von Tweets (auf } \\
\text { Richtigkeit selbst prüfen) }\end{array}$ & 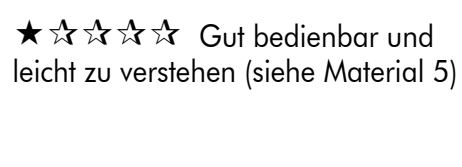 \\
\hline TrackMyHashtag (Demo) & $\begin{array}{l}\text { https://www.trackmyhash- } \\
\text { tag.com }\end{array}$ & 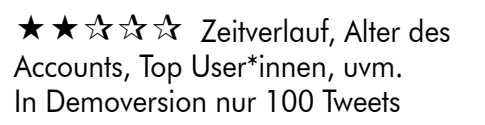 & 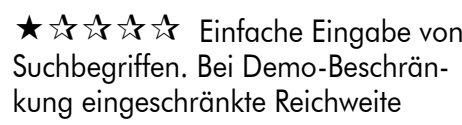 \\
\hline Sentiment Viz & $\begin{array}{l}\text { https://www.csc2.ncsu.edu/ } \\
\text { faculty/healey/tweet viz/ } \\
\text { tweet_app/ }\end{array}$ & $\begin{array}{l}\star \star \star\langle\downarrow \text { Visualisiert Tweets } \\
\text { in einer Sentiment Analyse als } \\
\text { Wortwolke, Heatmap, Affinitäten etc. }\end{array}$ & 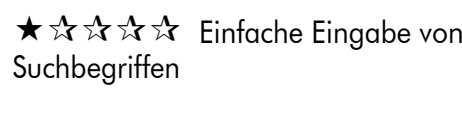 \\
\hline Tweeplers & https://www.tweeplers.com & $\begin{array}{l}\star \star \star \vec{\lambda} \text { Visualisiert und } \\
\text { rankt im Trend liegende Tweets oder } \\
\text { Hashtags z. B. nach Ländern }\end{array}$ & $\begin{array}{l}\star \star \star \forall \\
\text { als Einstieg nützlich. Andere Tools } \\
\text { weniger sinnvoll }\end{array}$ \\
\hline Social Bearing (Demo) & https://socialbearing.com/ & 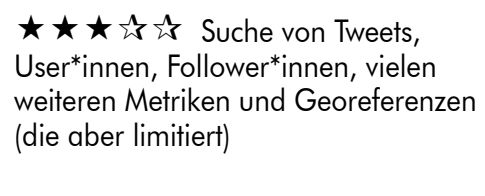 & $\begin{array}{l}\star \star \star \Delta \star \lambda \text { Suchworteingabe. } \\
\text { In der freien Version werden wenig } \\
\text { georeferenzierte Tweets angezeigt } \\
\text { und es wird eine Twitter-Anmeldung } \\
\text { benötigt. }\end{array}$ \\
\hline Social Searcher (Demo) & $\begin{array}{l}\text { https://www.social-searcher. } \\
\underline{\text { com/ }}\end{array}$ & $\begin{array}{l}\star \star \star \star \star \succ \text { Ergebnisse zu einem } \\
\text { Hashtag oder Suchbegriff werden } \\
\text { übersichtlich auf einer Pinnwand } \\
\text { angezeigt. Über "detailed statistics" } \\
\text { werden Sentiment Analysen, } \\
\text { Auswertungen nach Quelle oder } \\
\text { Uhrzeit oder Wortwolken verwandter } \\
\text { Hashtags angeboten. }\end{array}$ & $\begin{array}{l}\star \star \star \star \star \star * \text { Suchworteingabe. } \\
\text { Bedienung gewöhnungsbedürftig, } \\
\text { dann aber gut anwendbar } \\
\text { (siehe Material } 4 \text { ) }\end{array}$ \\
\hline Google MyMaps & $\begin{array}{l}\text { https://www.google.com/ } \\
\underline{\text { maps/d/?hl=de }}\end{array}$ & $\begin{array}{l}\star \star \star \star \star \succ \text { Eigene Karten } \\
\text { erstellen, zu denen jegliche Twitter- } \\
\text { Ereignisse eingefügt werden können }\end{array}$ & $\begin{array}{l}\star \star \star \star \star \text { Daten müssen vorher } \\
\text { bearbeitet und hochgeladen werden. } \\
\text { Einarbeitung notwendig }\end{array}$ \\
\hline
\end{tabular}




\section{Material 3: Reflexionsimpulse für die qualitative Auswertung von Twitter-Tweets}

\section{Quelle bewerten:}

- Von wem stammt der Tweet? Wie glaubwürdig sind die Angaben zur Person des/der Twitternutzer*in?

- Wie positioniert sich diese Person, welche Meinungen vertritt sie, welcher Gruppe (politische Strömung, Organisation, Firma, Verein, sozialer Gruppe etc.) gehört sie an?

- In welchem Zusammenhang steht diese Person oder Organisation zu anderen Nutzer*innen? Welchen Nutzer*innen folgt sie, von wem wird sie gefolgt?

\section{Informationsart und -güte bewerten:}

- Handelt es sich bei dem Inhalt des Tweets um Information, Meinung, Werbung, Beeinflussung oder Unterhaltung?

\section{Sprachliche Auswertung:}

- Welche Begriffe werden verwendet? Sind diese eher neutral gewählt oder suggestiv, offenbaren sie Meinung oder vertreten sie deutliche Positionen?

- An welche Debatten wird der Tweet angegliedert, z. B. über gewählte Hashtags?

\section{Material auswerten:}

- Was vermitteln die angehängten Fotos, Grafiken, Statistiken oder Videos?

- Welche Links werden gesetzt, in welche Zusammenhänge rücken sie den Tweet?

\section{Tweet-Resonanz bewerten:}

- Wer retweetet diesen Tweet?

- Wie wird der Tweet aufgenommen, wer antwortet darauf in welcher Art und Weise?

- Wie entwickelt sich die Debatte über diesen Tweet (in den Antworten)? 